\title{
USING CORRELATION AND DETERMINATION NUMBER OF FACTORS TO STUDY THE RESPONSE OF MAIZE THREE-WAY CROSSES TO ELEVATED NITROGEN FERTILIZER RATE
}

(Received: 15.2.2018)

\author{
By \\ M. B. A. El- Koomy, Maha M. G. Balba, A.K. Mostafa and *Hoda E.A. Ibrahim \\ Maize Research Department, Field crops Research Institute, * Center Laboratory For Design and \\ Statistical Analysis Research, Agricultural Research Center, Giza, Egypt
}

\begin{abstract}
Maize response to high nitrogenous fertilization rates is one of the means to know maximum productivity. Also genetic selection of maize hybrids is related to using high nitrogen levels, which require a strong $\mathrm{N}$ input to accomplish their yield potential. The present study aimed to investigate the response of maize three-way crosses to high nitrogen rates. The experiment was conducted at two locations, namely Gemmeiza and Mallawy, with split plot design in four replicates, during 2016 and 2017 seasons. At Gemmeiza the highest mean values of the number of rows/ear and grain yield fed ${ }^{-1}$ were 15.9 and 33.08 ard./ fed, respectively obtained by TWC353 hybrid when received $150 \mathrm{~kg} \mathrm{~N} / \mathrm{fed}$. Concerning Mallawy location, the highest mean values of the number of rows ear ${ }^{-1}$ and grain yield ardab fed $^{-1} 15.15$ and 33.04, respectively obtained by TWC353 hybrid when received 135 and $150 \mathrm{~kg}$ $\mathrm{N} /$ fed. Highly significant and positive associations were obtained among grain yield fed $^{-1}$ and each of plant height, ear height, ear length, and 100 kernel weight at Gemmeiza location. While at Mallawy location, grain yield had a positive and significant correlation with number of ears/plot ear length, kernels row $^{-1}$ and 100 kernel weight. Factor analysis classified the nine studied traits, as grain yield components, into three main factors explained $69.26 \%$ of the total variability in the dependent structure. At Gemmeiza location, Factor 1 was responsible for $27.26 \%$ of the total variation and included plant height, ear height and grain yield. Factor 2 included number of ears/plot. ear diameter, number of rows per ear and 100 kernel weight and contributed $25.29 \%$ of the total variation. Ear length and kernels per row of the factor3 accounted for $16.71 \%$ of the total variation. At Mallawy location, three factors explained $73.52 \%$ of the total variability in the dependence structure. Factor 1 included three variables which accounted for $28.17 \%$ of the total variability. The three variables were plant height, ear height and 100 kernel weight. The three variables had high communality with factor 1. Factor 2 was made up of the number of ears per plot, ear length, kernels per row and grain yield. The sign of the loading values indicates the direction of the relationship between the factor and its related traits. It accounted for $24.76 \%$ of the total variability in the dependence structure. Factor 3 was responsible for $20.584 \%$ of the total variability in the dependence structure. It included the two traits ear diameter and number of rows/ear.
\end{abstract}

Key words: Zea mays, N-rates, Grain yield traits, Correlation coefficients, Factor analysis.

\section{INTRODUCTION}

Nitrogen plays a significant role through various physiological operations and photosynthetic activities in maize. In maize the requirement of nitrogen depends on weather conditions, soil type and crop rotation pattern (Bundy et al., 2011 and Majid et al., 2017). Several researchers reported that nitrogen is the most limiting factor to increase maize grain yield and its components. They found that plant height, ear height, ear diameter, ear length, No. of rows ear ${ }^{-1}$, No. of grains row ${ }^{-1}$, ear weight, ear grain weight, 100-grain weight, shelling percentage, grain yield plant $^{-1}$ and grain yield fed $^{-1}$ of maize increased by increasing nitrogen level (Gouda et al., 1992; Gouda, 1997. Gouda et al., 2009 and Kandil, 2013).

The coefficient of correlation helps to measure the level of relationships between the traits and also establishes the level at which these traits are mutually different (Nagabhushan et al., 2011). 
There are several strategies to increase crop productivity, e.g. improving farming practices, employing merging technology, using modern and high yielding maize hybrids which more response to high rates of nitrogenous fertilizers. El-Badawy and Mehasen (2011) reported that application of 140 or $160 \mathrm{~kg}$ N/fed significantly increased all growth characters, yield and yield components of maize.

On the contrary, nitrogen deficiency decreased grain yield for all hybrids. However, there were no significant differences regarding harvest index, leaf area or plant weight at flowering stage between the $\mathrm{N}$-deficient and the control plants of all hybrids. Dry matter production after flowering of the nitrogen deficient plants was significantly lower than that of the control plants in all hybrids. Also, N deficiency accelerated senescence, i.e. decreased chlorophyll and protein contents after anthesis, more for the earlier released hybrids than for the later ones (Ding et al., 2005).

Nitrogen plays an essential role in many compounds which are essential for plant growth, chlorophyll synthesis and many enzymes. It is considered the key element in increasing crops productivity, and also, helps in the use of $\mathrm{P}, \mathrm{K}$ and other elements in plants.

Additionally, Atia and Mahmoud (2006) found that the highest grain yield (ard/fed) of the tested cultivar was produced by supplying 160 $\mathrm{kg} \mathrm{N} / \mathrm{fed}$. Majid et al.(2017) found that the highest grain yield was obtained from the plot treated with ( $345 \mathrm{~kg} \mathrm{~N} / \mathrm{fed})$

These correlations also give reliable and useful information on nature, extent and direction of selection. Eraky et al. (1982), ElBadawy and Mehasen (2011) and Nzuve et al. (2014) reported that grain yield $\mathrm{fed}^{-1}$ of maize was highly positively correlated with ear length, the number of grains /row, ear diameter, shelling\%, grain yield plant ${ }^{-1}$ and 100-kernel weight but positively and significantly correlated with both of plant height and ear height.

Factor analysis is a type of multivariate analysis that can be used to reduce a large number of correlated variables to a minimum number of independent main factors. Bharathiveeramani and Prakash (2012) found that sixteen factors were extracted using principal component analysis as extraction method. Out of the 16, only 5 factors had eigen value greater than one, and these factors alone accounted for $78.3 \%$ of the total variance. The loadings of each variable onto each factor was analysed from the rotated factor matrix obtained through varimax rotation. First factor accounted for $22.93 \%$ of the total variation and showed significant associations of traits related to duration, namely days to $50 \%$ tasseling, days to $50 \%$ silking, anthesis silking interval and days to maturity. Second factor, with $19.22 \%$ of the total variation, had higher loadings of plant height and ear height which are the important growth traits in maize. Factor 3 with a contribution of $17.91 \%$ to the total variation was found to be significantly associated with the important yield traits viz. cob yield/plant, grain yield/plant and ear girth. Factor 4 (9.99\% variance) and factor 5 (8.33\% variance) were loaded mainly with hundred seed weight and grain setting length respectively.

The current study aimed to investigate the response of four 3-way crosses of maize to elevated nitrogen fertilizer and determine the dependence relationships between grain yield and its components by using two statistical procedures including simple correlation and factor analysis.

\section{MATERIALS AND METHODS}

A field trial was conducted at two locations, i.e., Gemmeiza and Mallawy Agric. Res. Stns., Field Crops Res. Inst., Agric. Res. Center, during two growing seasons 2016 and 2017 to study the response of four Maize 3-way crosses to increased nitrogen fertilizer rate.The experimental design was split plot with four replications. Nitrogen treatments were assigned to the main plots with three levels including 120 , 135 and $150 \mathrm{~kg} \mathrm{~N} / \mathrm{fed}$ with three-way crosses TWC 324, TWC 329,TWC 353 and TWC 354 arranged in the sub plots. The experimental unit was 4 rows, $6 \mathrm{~m}$ in length, $80 \mathrm{~cm}$ in width, and $20 \mathrm{~cm}$ between hills. One blank row was left between plots. All plants in the $2^{\text {nd }}$ and $3^{\text {rd }}$ rows were harvested and grain yield was adjusted to $15.5 \%$ moisture. Phosphorus at a rate of $30 \mathrm{~kg}$ $\mathrm{P}_{2} \mathrm{O}_{5}$ fed $^{-1}$ in the form of Superphosphate $(15 \%$ $\mathrm{P}_{2} \mathrm{O}_{5}$ ) and Potassium at a rate of $24 \mathrm{~kg} \mathrm{~K} \mathrm{~K}_{2} \mathrm{O}$ per fed in the form of Potassium sulphate $48 \% \mathrm{~K}_{2} \mathrm{O}$ were added before planting. Soil samples at $(0-$ $30 \mathrm{~cm}$ depth) were taken from the experimental site before planting for physical and chemical analysis. This study was performed in Mallawy (clay loam soil) and Gemmeiza (clay loam soil). Soil properties are presented in Table (1) according to Page et al. (1982). All other 
Table (1): Physical and chemical analysis of both experimental sites (Mallawy and Gemmeiza).

\begin{tabular}{|c|c|c|c|c|c|}
\hline \multirow[t]{2}{*}{ Soil characters } & \multicolumn{2}{|c|}{ Physical analysis } & \multirow[t]{3}{*}{ Soil characters } & \multicolumn{2}{|c|}{ Chemical analysis } \\
\hline & Mallawy & Gemmeiza & & Mallawy & Gemmeiza \\
\hline Sand\% & 22.4 & 20.4 & & & \\
\hline Silt \% & 37.0 & 32.8 & Organic matter \% & 0.89 & 0.79 \\
\hline Clay \% & 40.5 & 46.8 & Total soluble N $\left(\mathrm{mg} \mathrm{kg}^{-1}\right)$ & 60.60 & 88.45 \\
\hline \multirow[t]{2}{*}{ Soil texture } & \multirow[t]{2}{*}{ Clay loam } & \multirow[t]{2}{*}{ Clay loam } & Available P (mg kg $\left.{ }^{-1}\right)$ & 6.15 & 8.20 \\
\hline & & & Available K (mg kg$\left.{ }^{-1}\right)$ & 293.5 & 305.0 \\
\hline
\end{tabular}

cultural practices were applied as recommended.

\subsection{Data recorded at harvest}

Data recorded for maize hybrids for both tested seasons were plant height $(\mathrm{cm})$, ear height $(\mathrm{cm})$, number of ears /plot, grain yield ( $\operatorname{ard} / \mathrm{fed})$, ear length $(\mathrm{cm})$, ear diameter $(\mathrm{cm})$, number of rows/ear, number of kernels /row, and 100kernel weight/ear $(\mathrm{g})$

\subsection{Statistical analysis}

A combined analysis of variance was computed across the two seasons according to Snedecor and Cochran (1989). On the other hand, Levene test (1960) was used to satisfy the assumption of homogeneity of variances before running the combined analysis. In this research, several statistical analyses were used in order to show the presence or absence of correlations among different dependent variables of maize hybrids. Variance analysis was applied; the simple correlation coefficients were computed among yield $\mathrm{fed}^{-1}$ and its components (Gomez and Gomez, 1984).

Factor analysis was applied according to Cattell (1965) to reduce a large number of correlated variables to a much smaller number of independent clusters of variables called factors. After the loading of the first factor was calculated, the process was repeated on the residuals matrix to find further factors. When the contribution of a factor to the total percentage of the trace was less than $10 \%$, the process was stopped. After extraction, the matrix of factor loadings was submitted to a Varimax Orthogonal Rotation, as applied by Kaiser (1958). The purpose of rotation was to rebuilding the larger loadings in each factor and to suppress the minor loading coefficient so as to improve the opportunity of achieving meaningful biological interpretation of each factor.

Analysis of variance of data and mean comparison of them was done using MSTATC and SPSS programs. Mean comparison was done using Least significant differences test at 5\% level of probability.

\section{RESULTS AND DISCUSSION}

\subsection{Combined analysis of variance}

Table (2) showed the combined analysis of variance for all studied traits. Test of homogeneity revealed that the error variance for the two seasons was homogenous, therefore combined analysis was processed. Year's mean squares were highly significant and significant for all the studied traits, except for ear length, number of row ear ${ }^{-1}$ and kernel weight at Mallawy location and for number of kernel /row in both sites. The hybrids mean squares were highly significant for all traits at two locations. $\mathrm{N}$ fertilizer level mean squares were nonsignificant for all studied traits in two locations, except for plant height, number of ears /plot and grain yield at Gemmeiza location. The interaction between years and hybrids mean squares was significant for all of the studied traits. The interaction between years and $\mathrm{N}$ levels mean squares were significant for number of ears/plot and 100 kernel weight traits at Gemmeiza location. The significant differences recorded for the different traits among the studied hybrids implied that maize hybrids included in this study had diverse genetic backgrounds (Nzuve et al., 2014 and Majid et al., 2017).

\subsection{Hybrids performance}

The results in Table (3) showed that the four evaluated hybrids significantly varied in all traits under study at two locations except number of ears /plot, number of row/ear and ear diameter at Mallawy location. This suggests that sufficient genetic variability exists in the genotypes which selected for study. TWC 324 followed by TWC 329 hybrids gave the highest values of plant height, ear height, ear length, kernels row $^{-1}$ and grain yield at Gemmeiza and Mallawy locations.While, TWC 353 recorded the highest means of ear diameter and No. rows/ear at the two locations. Meanwhile, TWC 354 recorded 
Table (2): Mean square values and significance for maize yield and yield attributes at two locations combined over two seasons.

\begin{tabular}{|c|c|c|c|c|c|c|c|}
\hline \multirow[t]{2}{*}{ S.O. V } & \multirow[t]{2}{*}{ df } & \multicolumn{2}{|c|}{ PHT } & \multicolumn{2}{|c|}{ EHT } & \multicolumn{2}{|c|}{ N E/P } \\
\hline & & Gemmeiza & Mallawy & Gemmeiza & Mallawy & Gemmeiza & Mallawy \\
\hline Years & 1 & $12489.84^{* *}$ & $4973.76 * *$ & $3626.042^{* * *}$ & $1120.67 *$ & $100.04^{* * *}$ & $2646.0^{* * *}$ \\
\hline $\mathbf{R}(\mathbf{Y})$ & 6 & $353.73 * *$ & 676.61* & $134.549^{\mathrm{NS}}$ & $303.38^{\mathrm{NS}}$ & $2.24^{\mathrm{NS}}$ & $23.25^{\mathrm{NS}}$ \\
\hline $\mathbf{N}$ & 2 & $386.19 *$ & $237.32^{\mathrm{NS}}$ & $79.17^{\mathrm{NS}}$ & $101.39^{\mathrm{NS}}$ & $22.59^{*}$ & $0.97^{\mathrm{NS}}$ \\
\hline NY & 2 & $33.59^{\mathrm{NS}}$ & $8.51^{\mathrm{NS}}$ & $29.17^{\mathrm{NS}}$ & $1.76^{\mathrm{NS}}$ & $24.51^{*}$ & $13.78^{\mathrm{NS}}$ \\
\hline Error(a) & 12 & 61.29 & 226.93 & 66.32 & 135.18 & 3.66 & 12.04 \\
\hline Hybr & 3 & $9106.51 * *$ & $7936.57^{* * *}$ & $3161.81^{* *}$ & $3077.90^{* * *}$ & $6.85^{*}$ & $5.69^{\mathrm{NS}}$ \\
\hline HY & 3 & $1677.34 * *$ & 994.71* & $813.24^{*}$ & $61.97^{\mathrm{NS}}$ & $14.46^{* *}$ & $8.53^{\mathrm{NS}}$ \\
\hline HXN & 6 & $467.45^{* *}$ & $663.55^{* *}$ & $272.22^{* *}$ & $443.49^{* *}$ & $1.52^{\mathrm{NS}}$ & $5.66^{\mathrm{NS}}$ \\
\hline HXNXY & 6 & $648.18 * *$ & $192.04^{\mathrm{NS}}$ & $450.69^{* *}$ & $165.82^{\mathrm{NS}}$ & $1.47^{\mathrm{NS}}$ & $3.14^{\mathrm{NS}}$ \\
\hline Error (b) & 54 & 90.94 & 221.56 & 77.49 & 125.43 & 2.35 & 4.73 \\
\hline \multirow[t]{2}{*}{ S.O. V } & \multirow[t]{2}{*}{ df } & \multicolumn{2}{|c|}{ EL } & \multicolumn{2}{|c|}{ ED } & \multicolumn{2}{|c|}{ RPE } \\
\hline & & Gemmeiza & Mallawy & Gemmeiza & Mallawy & Gemmeiza & Mallawy \\
\hline Years & 1 & $26.25^{* *}$ & 0.042 & $5.90^{* * *}$ & $0.57^{* *}$ & $9.004^{* * *}$ & $1.307^{\mathrm{NS}}$ \\
\hline $\mathbf{R}(\mathbf{Y})$ & 6 & $5.83^{* *}$ & $8.793^{*}$ & $0.07 *$ & $0.25^{* * *}$ & $0.953^{\mathrm{NS}}$ & $1.627^{\mathrm{NS}}$ \\
\hline $\mathbf{N}$ & 2 & $0.30^{\mathrm{NS}}$ & $2.21^{\mathrm{NS}}$ & $0.02^{\mathrm{NS}}$ & $0.01^{\mathrm{NS}}$ & $0.136^{\mathrm{NS}}$ & $1.080^{\mathrm{NS}}$ \\
\hline NY & 2 & $1.29^{\mathrm{NS}}$ & $0.405^{\mathrm{NS}}$ & $0.18^{\mathrm{NS}}$ & $0.12^{\mathrm{NS}}$ & $0.354^{\mathrm{NS}}$ & $1.718^{\mathrm{NS}}$ \\
\hline Error(a) & 12 & 1.14 & 2.863 & 0.02 & 0.05 & 0.506 & 1.376 \\
\hline Hybr & 3 & $27.36^{* * 1}$ & $22.78^{* * *}$ & $0.08^{*}$ & $0.012^{\mathrm{NS}}$ & $24.973^{* * 4}$ & $2.837^{\mathrm{NS}}$ \\
\hline HY & 3 & $2.24^{\mathrm{NS}}$ & $3.739^{\mathrm{NS}}$ & $0.07 *$ & $0.004^{\mathrm{NS}}$ & $1.099^{\mathrm{NS}}$ & $2.591^{\mathrm{NS}}$ \\
\hline HXN & 6 & $0.93^{\mathrm{NS}}$ & $3.05^{\mathrm{NS}}$ & $0.01^{\mathrm{NS}}$ & $0.019^{\mathrm{NS}}$ & $0.646^{\mathrm{NS}}$ & $0.609^{\mathrm{NS}}$ \\
\hline HXNXY & 6 & $1.81^{\mathrm{NS}}$ & $3.06^{\mathrm{NS}}$ & $0.01^{\mathrm{NS}}$ & $0.152^{* *}$ & $0.671^{\mathrm{NS}}$ & $2.276^{\mathrm{NS}}$ \\
\hline Error (b) & 54 & 1.85 & 1.43 & 0.02 & 0.048 & 0.837 & 1.420 \\
\hline \multirow[t]{2}{*}{ S.O. V } & df & \multicolumn{2}{|c|}{ KPR } & \multicolumn{2}{|c|}{ KWT } & \multicolumn{2}{|c|}{ YIELD } \\
\hline & & Gemmeiza & Mallawy & Gemmeiza & Mallawy & Gemmeiza & Mallawy \\
\hline Years & 1 & $0.001^{\mathrm{NS}}$ & $6.30^{\mathrm{NS}}$ & $182.88^{* * *}$ & $24.00^{\mathrm{NS}}$ & $31.020^{*}$ & 1377.59 ** \\
\hline $\mathbf{R}(\mathbf{Y})$ & 6 & $21.01^{\mathrm{NS}}$ & $15.48^{\mathrm{NS}}$ & $0.69^{\mathrm{NS}}$ & $32.58^{*}$ & $10.785^{\mathrm{NS}}$ & $25.29^{\mathrm{NS}}$ \\
\hline $\mathbf{N}$ & 2 & $16.032^{\mathrm{NS}}$ & $12.99^{\mathrm{NS}}$ & $2.04^{\mathrm{NS}}$ & $6.77^{\mathrm{NS}}$ & $198.627^{* *}$ & $96.23 *$ \\
\hline NY & 2 & $16.85^{\mathrm{NS}}$ & $3.65^{\mathrm{NS}}$ & $15.14^{* *}$ & $7.24^{\mathrm{NS}}$ & $11.121^{\mathrm{NS}}$ & $6.93^{\mathrm{NS}}$ \\
\hline Error(a) & 12 & 9.73 & 6.99 & 0.73 & 7.07 & 4.683 & 21.87 \\
\hline Hybr & 3 & $102.22^{* *}$ & $47.28 * *$ & $49.36^{* *}$ & $113,95^{* * *}$ & $222.244 * *$ & $63.43^{* *}$ \\
\hline HY & 3 & $8.78^{\mathrm{NS}}$ & $10.31^{\mathrm{NS}}$ & $18.35^{* *}$ & $7.39^{\mathrm{NS}}$ & $50.269^{* *}$ & $17.07^{\mathrm{NS}}$ \\
\hline HXN & 6 & $18.93^{* * *}$ & $10.71^{\mathrm{NS}}$ & $8.95^{* * *}$ & $20.77^{*}$ & $11.761 *$ & $33.51 *$ \\
\hline HXNXY & 6 & $16.32^{* * *}$ & $5.25^{\mathrm{NS}}$ & $7.30^{* * *}$ & $7.92^{\mathrm{NS}}$ & $17.123 * *$ & $21.5^{\mathrm{NS}}$ \\
\hline Error (b) & 54 & 3.71 & 9.13 & 1.63 & 6.69 & 4.90 & 12.59 \\
\hline
\end{tabular}

$*$ and ** are significant at $0.05 \%$ and $0.01 \%$ level of probability plant height (PHT), ear height (EHT), number of ears /plot (NE/P), ear length (EL), ear diameter (ED), number of row ear ${ }^{-1}$ (RPE), number of kernels per row (KPR), 100 kernels weight (KWT), and grain yield (ardab /fed ).

the highest means of the number of ears per plot and kernels /row. The 4 tested maize hybrids showed some significant differences in ear length, 100 kernel weight and yield combined over the two years. Superiorities of these traits $\left(21.63 \mathrm{~cm}, 38.35 \mathrm{~g}\right.$ and $\left.31.29 \mathrm{ardfed}^{-1}\right)$ were recorded by TWC 324 followed by TWC 329 hybrid $\left(20.78 \mathrm{~cm}, 37.12 \mathrm{~g}\right.$ and $\left.27.66 \mathrm{ardfed}^{-1}\right)$ at Gemmeiza location. While for Mallawy location, the highest values of ear length, 100 kernel weight and grain yield were recorded by TWC $324\left(21.83 \mathrm{~cm}, 40.65 \mathrm{~g}\right.$ and $\left.29.97 \mathrm{ardfed}^{-1}\right)$, followed by TWC $329(20.34 \mathrm{~cm}, 39.53 \mathrm{~g}$ and 28.76 ardfed $^{-1}$ ). The hybrid TWC353 gave the highest values of ear diameter (4.88 and $5.15 \mathrm{~cm})$, Number of rows/ear (15.94 and 14.70) at Gemmeiza and Mallawy location, respectively. The results of varietal differences in yield and yield attributes of maize hybrids in this study are in agreement with those obtained 
Table (3): Mean performance of three- way maize hybrids at Gemmeiza and Mallawy across years (combined analysis).

\begin{tabular}{|l|l|c|c|c|c|c|c|c|c|c|}
\hline Locations & Hybrids & PHT & EHT & NE/P & EL & ED & RPE & KPR & KWT & Yield \\
\hline \multirow{4}{*}{ Gemmeiza } & TWC324 & 270.83 & 153.96 & 54.21 & 21.63 & 4.76 & 13.54 & 46.36 & 38.35 & 31.29 \\
\cline { 2 - 10 } & TWC329 & 273.96 & 157.50 & 53.67 & 20.78 & 4.85 & 14.17 & 44.30 & 37.12 & 27.66 \\
\cline { 2 - 10 } & TWC353 & 237.92 & 133.75 & 53.96 & 19.07 & 4.88 & 15.94 & 41.90 & 35.63 & 24.01 \\
\cline { 2 - 10 } & TWC354 & 239.58 & 138.96 & 54.92 & 20.48 & 4.78 & 14.37 & 46.13 & 35.22 & 26.36 \\
\hline \multirow{5}{*}{ Mallawy } & LSD at 0.05 \% & $\mathbf{5 . 5 2}$ & $\mathbf{5 . 0 9}$ & $\mathbf{0 . 8 8}$ & $\mathbf{0 . 7 8}$ & $\mathbf{0 . 0 8}$ & $\mathbf{0 . 5 2}$ & $\mathbf{1 . 1 1}$ & $\mathbf{0 . 7 3}$ & $\mathbf{1 . 2 8}$ \\
\hline & TWC324 & 240.54 & 129.67 & 54.58 & 21.83 & 5.12 & 13.90 & 43.28 & 40.65 & 29.97 \\
\cline { 2 - 10 } & TWC329 & 243.25 & 129.96 & 53.63 & 20.34 & 5.15 & 14.37 & 40.73 & 39.53 & 28.86 \\
\cline { 2 - 10 } & TWC353 & 210.25 & 109.46 & 53.50 & 19.64 & 5.15 & 14.70 & 40.11 & 35.68 & 26.11 \\
\cline { 2 - 10 } & TWC354 & 210.67 & 111.0 & 53.79 & 19.90 & 5.12 & 14.11 & 40.77 & 37.70 & 28.19 \\
\hline & LSD at $\mathbf{0 . 0 5} \%$ & $\mathbf{8 . 6 1}$ & $\mathbf{6 . 4 8}$ & $\mathbf{N S}$ & $\mathbf{0 . 6 9}$ & $\mathbf{N S}$ & $\mathbf{N S}$ & $\mathbf{1 . 7 4}$ & $\mathbf{1 . 4 9}$ & $\mathbf{2 . 0 5}$ \\
\hline
\end{tabular}

plant height(PHT), ear height (EHT) number of ears /plot(NE/P), ear length (EL), ear diameter (ED), number of row /ear (RPE), number of kernels /row (KPR), 100 kernel weight (KWT), and grain yield (ardab /fed ).

by Gouda et al., (1992), Gouda et al.,(2009), Mehasen and El-Gizawy (2010) and El-Badawy and Mehasen (2011).

\subsection{Effect of $\mathrm{N}$ rates}

Results presented in Table (4) show that the increase in $\mathrm{N}$-rate caused an increase in yield and yield traits of maize in the combined analysis by increasing $\mathrm{N}$-level up to $150 \mathrm{~kg} \mathrm{~N}$ carbohydrates which are converted to proteins and to protoplasm while the small proportion left available for cell wall material. Furthermore, the increase in grain yield $\mathrm{fed}^{-1}$ is attributed mainly to the increases in yield components. These results are in harmony with those of Atia and Mahmoud (2006), Gouda et al. (2009), Kandil (2013), Mitiku and Asnakech (2016).

Table (4): Effect of nitrogen fertilization levels on grain yield and its traits of maize hybrids at Gemmeiza and Mallawy locations over two seasons.

\begin{tabular}{|l|l|l|l|l|l|l|l|l|l|l|}
\hline Locations & Hybrids & PHT & EHT & NE/P & EL & ED & RPE & KPR & KWT & Y \\
\hline \multirow{4}{*}{ Gemmeiza } & $120 \mathrm{~kg} \mathrm{~N}$ & 251.56 & 144.38 & 53.66 & 20.42 & 4.80 & 14.54 & 44.73 & 36.31 & 25.15 \\
\cline { 2 - 11 } & $135 \mathrm{~kg} \mathrm{~N}$ & 257.66 & 147.50 & 53.75 & 20.60 & 4.84 & 14.55 & 45.35 & 36.81 & 26.81 \\
\cline { 2 - 11 } & $150 \mathrm{~kg} \mathrm{~N}$ & 257.50 & 146.25 & 55.16 & 20.45 & 4.80 & 14.43 & 43.94 & 36.61 & 30.05 \\
\hline \multirow{5}{*}{ Mallawy } & LSD at 0.05 \% & $\mathbf{4 . 2 6 4}$ & NS & $\mathbf{1 . 0 4 3}$ & NS & NS & NS & NS & NS & $\mathbf{1 . 1 7}$ \\
\cline { 2 - 11 } & $120 \mathrm{~kg} \mathrm{~N}$ & 225.22 & 120.28 & 54.03 & 20.21 & 5.13 & 14.22 & 40.55 & 37.95 & 26.69 \\
\cline { 2 - 11 } & $135 \mathrm{~kg} \mathrm{~N}$ & 224.06 & 118.13 & 53.69 & 20.72 & 5.12 & 14.48 & 41.29 & 38.86 & 28.03 \\
\cline { 2 - 10 } & $150 \mathrm{~kg} \mathrm{~N}$ & 229.25 & 121.66 & 53.91 & 20.36 & 5.14 & 14.12 & 41.82 & 38.36 & 30.14 \\
\hline & LSD at 0.05 \% & NS & NS & NS & NS & NS & NS & NS & NS & $\mathbf{2 . 5 5}$ \\
\hline
\end{tabular}

plant height(PHT), ear height (EHT), number of ears /plot(NE/P), ear length (EL), ear diameter (ED), number of row/ear (RPE), number of kernels/row (KPR), 100 kernel weight (KWT), and grain yield (ardab /fed ).

/fed. The highest values of plant height, ear height, ear length, ear diameter, number of rows ear ${ }^{-1}$, and number of kernels ear ${ }^{-1}$ and 100 kernel weight were obtained with fertilizer level of $135 \mathrm{~kg} \mathrm{~N} /$ fed. Increasing N-rates to $150 \mathrm{~kg} \mathrm{~N} / \mathrm{fed}$ led to increases in grain yield fed $^{-1}$ at Gemmeiza location, while for Mallawy location, the highest values of ear diameter and grain yield were obtained by $135 \mathrm{~kg}$ nitrogen, while $150 \mathrm{~kg}$ nitrogen gave the highest values of plant height, ear height, kernels/row and grain yield. Russell (1973) stated that the higher the nitrogen supplies the more rapidly the synthesized

\subsection{Effect of interaction}

At Gemmeiza location, the interaction between maize hybrids and $\mathrm{N}$ fertilization levels Table (5) had a significant effect on plant height, ear height, 100 kernel weight and grain yield $\operatorname{ardabfed}^{-1}$ across two years. The highest values of plant height $(282.50 \mathrm{~cm})$ and ear height $(163.13 \mathrm{~cm})$ were obtained by TWC 324 hybrid when received $150 \mathrm{~kg} \mathrm{~N}$ fed $^{-1}$, while the lowest value of plant height $(235 \mathrm{~cm})$ was obtained by TWC 354 hybrid under $135 \mathrm{~kg} \mathrm{~N} / \mathrm{fed}$. The highest mean values of the number of row sear ${ }^{-1}$ and grain yield ardab fed ${ }^{-1}$ were 15.9 and 33.08 
$\operatorname{ard} / \mathrm{fed}^{-1}$ (Fig. 1), respectively obtained by TWC353 hybrid when received $150 \mathrm{~kg} \mathrm{~N} / \mathrm{fed}$. Whereas, the lowest values of kernelsrow ${ }^{-1}$ and grain yield fed $^{-1}$ were 40.28 and 20.84 ardab/feddan, respectively obtained by TWC353 and TWC324 hybrid under $150 \mathrm{~kg}$ N/fed similar results were reported by Eraky et al. (1982), Gouda,1997 and Gouda et al., 2009.

Concerning Mallawy location the highest values of plant height $(250.13 \mathrm{~cm})$ and ear height $(138.13 \mathrm{~cm})$ were obtained by TWC 329 hybrid when received $135 \mathrm{~kg} \mathrm{~N} / \mathrm{fed}$, while the lowest
Values of plant height $(197.38 \mathrm{~cm})$ were obtained by TWC 353 hybrid under $135 \mathrm{~kg} \mathrm{~N} /$ fed. The highest mean values of the number of row/ear and grain yield $\mathrm{fed}^{-1}$ were 15.15 and 33.04 ard./fed.(Fig. 2), respectively, obtained by TWC353 hybrid when received 135 and $150 \mathrm{~kg}$ $\mathrm{N} /$ fed.Whereas, the lowest values of kernels/row and grain yield $\mathrm{fed}^{-1}$ were 39.10 and 25.01 $\operatorname{ardabfeddan}^{-1}$, obtained by TWC329 and TWC324 hybrid under 120 and $135 \mathrm{~kg} \mathrm{~N} / \mathrm{fed}$. These results are in agreement with the findings of Gouda et al. (1992) and Akmal et al. (2010).

Table (5): Effect of maize hybrids and $\mathbf{N}$ fertilization levels interaction on grain yield and some of its attributes in combined analysis.

\begin{tabular}{|c|c|c|c|c|c|c|c|c|c|c|}
\hline \multirow{3}{*}{ Hybrids } & \multirow{3}{*}{ Locations } & \multicolumn{3}{|c|}{ PHT } & \multicolumn{3}{|c|}{ EHT } & \multicolumn{3}{|c|}{$\mathrm{NE} / \mathrm{P}$} \\
\hline & & \multicolumn{9}{|c|}{ N level ${ }^{-1}$} \\
\hline & & 120 & 135 & 150 & 120 & 135 & 150 & 120 & 135 & 150 \\
\hline \multirow[t]{2}{*}{ TWC324 } & Gemmeiza & 255.00 & 282.50 & 275.00 & 146.88 & 163.13 & 151.88 & 54.00 & 53.25 & 55.38 \\
\hline & Mallawy & 238.38 & 240.13 & 243.13 & 131.13 & 127.25 & 130.63 & 55.37 & 53.25 & 55.13 \\
\hline \multirow[t]{2}{*}{ TWC329 } & Gemmeiza & 273.75 & 275.63 & 272.50 & 159.38 & 160.0 & 153.13 & 53.25 & 53.63 & 54.13 \\
\hline & Mallawy & 230.50 & 250.13 & 249.13 & 119.0 & 138.13 & 132.75 & 53.62 & 54.37 & 52.88 \\
\hline \multirow[t]{2}{*}{ TWC353 } & Gemmeiza & 236.88 & 237.50 & 239.38 & 135.00 & 130.00 & 136.25 & 53.38 & 53.63 & 54.88 \\
\hline & Mallawy & 221.37 & 197.38 & 212.0 & 116.13 & 102.37 & 109.88 & 53.50 & 53.00 & 54.00 \\
\hline \multirow{2}{*}{ TWC354 } & Gemmeiza & 240.63 & 235.00 & 243.13 & 136.25 & 136.88 & 143.75 & 54.00 & 54.50 & 56.25 \\
\hline & Mallawy & 210.63 & 208.63 & 212.75 & 114.88 & 104.75 & 113.3 & 53.62 & 54.13 & 53.63 \\
\hline \multirow{2}{*}{$\begin{array}{c}\text { LSD } \\
\text { at } 0.05\end{array}$} & Gemmeiza & \multicolumn{3}{|c|}{9.56} & \multicolumn{3}{|c|}{8.82} & \multicolumn{3}{|c|}{ NS } \\
\hline & Mallawy & \multicolumn{3}{|c|}{14.92} & \multicolumn{3}{|c|}{11.23} & \multicolumn{3}{|c|}{ NS } \\
\hline \multirow{3}{*}{ Hybrids } & \multirow{3}{*}{ Locations } & \multicolumn{3}{|c|}{ EL } & \multicolumn{3}{|c|}{ ED } & \multirow{2}{*}{\multicolumn{3}{|c|}{ RPE }} \\
\hline & & \multicolumn{8}{|c|}{$\mathrm{N}$ level $^{-1}$} & \\
\hline & & 120 & 135 & 150 & 120 & 135 & 150 & 120 & 135 & 150 \\
\hline \multirow[t]{2}{*}{ TWC324 } & Gemmeiza & 21.60 & 21.53 & 21.68 & 4.7 & 4.82 & 4.75 & 13.48 & 13.35 & 13.80 \\
\hline & Mallawy & 21.30 & 22.05 & 22.13 & 5.03 & 5.15 & 5.15 & 13.75 & 14.40 & 13.55 \\
\hline TWC329 & Gemmeiza & 20.93 & 21.05 & 20.38 & 4.85 & 4.9 & 4.8 & 14.19 & 14.58 & 13.75 \\
\hline & Mallawy & 19.75 & 20.55 & 20.73 & 5.18 & 5.13 & 5.15 & 14.45 & 14.25 & 14.40 \\
\hline TWC353 & Gemmeiza & 19.13 & 19.15 & 18.93 & 4.88 & 4.88 & 4.87 & 15.91 & 16.01 & 15.90 \\
\hline & Mallawy & 20.25 & 20.08 & 18.60 & 5.18 & 5.13 & 5.15 & 14.52 & 15.15 & 14.42 \\
\hline TWC354 & Gemmeiza & 19.93 & 20.68 & 20.83 & 4.78 & 4.78 & 4.78 & 14.58 & 14.26 & 14.28 \\
\hline & Mallawy & 19.52 & 20.20 & 20.00 & 5.15 & 5.07 & 5.13 & 14.15 & 14.10 & 14.42 \\
\hline LSD & Gemmeiza & & NS & & & NS & & & NS & \\
\hline & Mallawy & & NS & & & NS & & & NS & \\
\hline & & & KPR & & & KWT & & & $\mathbf{Y}$ & \\
\hline Hybrids & Locations & & & & & level ${ }^{-1}$ & & & & \\
\hline & & 120 & 135 & 150 & 120 & 135 & 150 & 120 & 135 & 150 \\
\hline TWC324 & Gemmeiza & 47.48 & 45.05 & 46.55 & 38.71 & 38.41 & 37.91 & 28.74 & 26.77 & 20.84 \\
\hline & Mallawy & 41.88 & 42.90 & 45.05 & 39.90 & 42.17 & 39.90 & 27.19 & 25.01 & 26.86 \\
\hline TWC329 & Gemmeiza & 42.75 & 45.40 & 44.75 & 36.30 & 37.32 & 37.74 & 24.24 & 32.06 & 26.79 \\
\hline & Mallawy & 39.10 & 41.57 & 41.50 & 38.12 & 39.97 & 40.50 & 27.71 & 29.68 & 29.77 \\
\hline TWC353 & Gemmeiza & 42.48 & 42.95 & 40.28 & 36.20 & 36.40 & 34.28 & 23.30 & 25.05 & 33.08 \\
\hline & Mallawy & 40.63 & 40.47 & 39.22 & 37.75 & 34.80 & 34.50 & 25.99 & 26.64 & 33.04 \\
\hline TWC354 & Gemmeiza & 46.20 & 48.00 & 44.18 & 34.04 & 35.13 & 36.50 & 29.43 & 27.88 & 29.78 \\
\hline & Mallawy & 40.60 & 40.20 & 41.50 & 36.02 & 38.52 & 38.57 & 31.80 & 25.48 & 30.21 \\
\hline & Gemmeiza & & 1.93 & & & 1.28 & & & 2.22 & \\
\hline at 0.05 & Mallawy & & NS & & & 2.59 & & & 3.59 & \\
\hline
\end{tabular}

plant height(PHT), ear height (EHT), number of ears /plot(NE/P), ear length (EL), ear diameter (ED), number of row /ear (RPE), number of kernels/row (KPR), 100 kernels weight (KWT), and grain yield (ardab /fed ). 


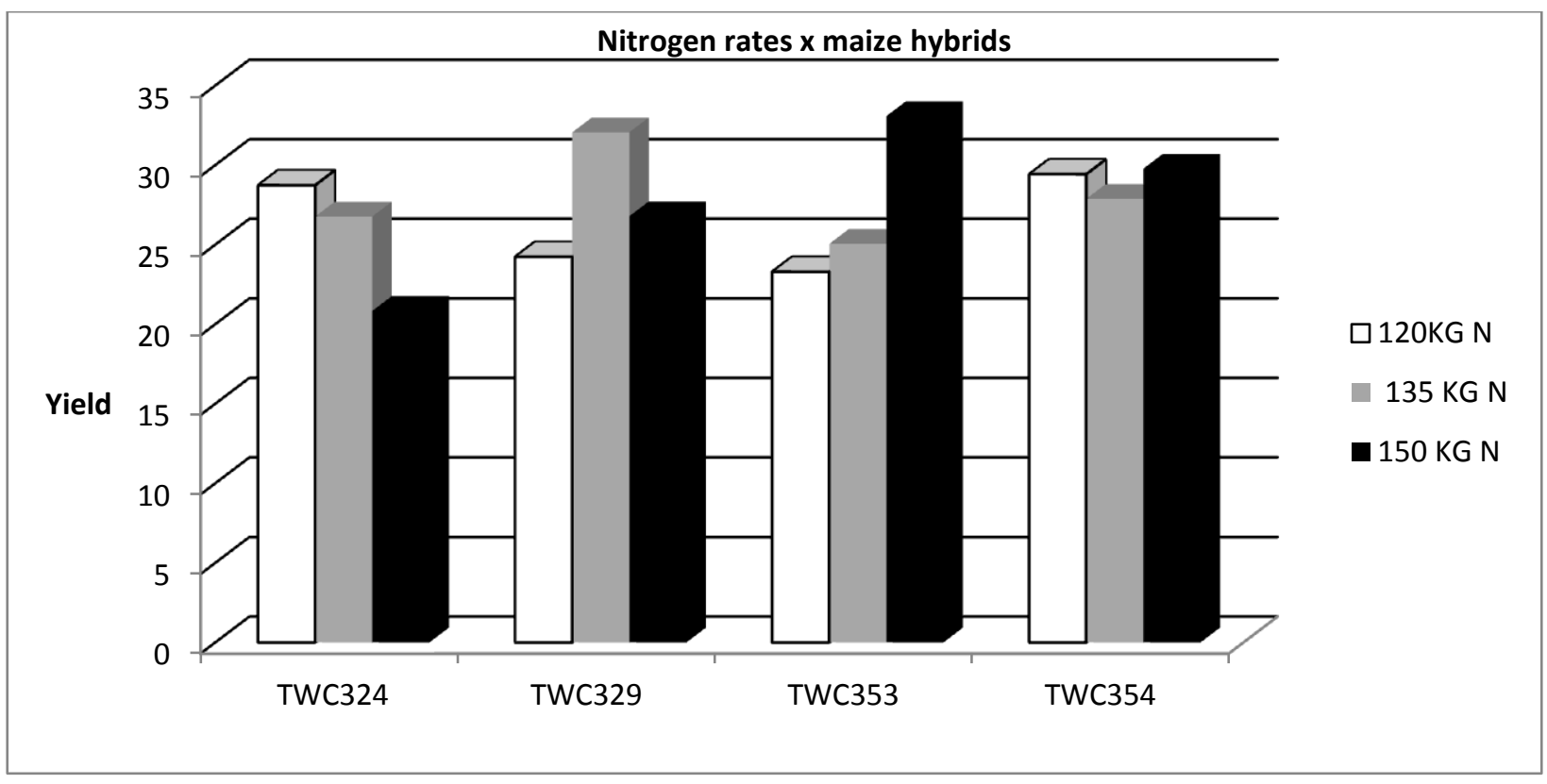

Fig. (1): Effect of interaction between nitrogen fertilization and maize hybrids on grain yield at Gemmeiza location over two seasons.

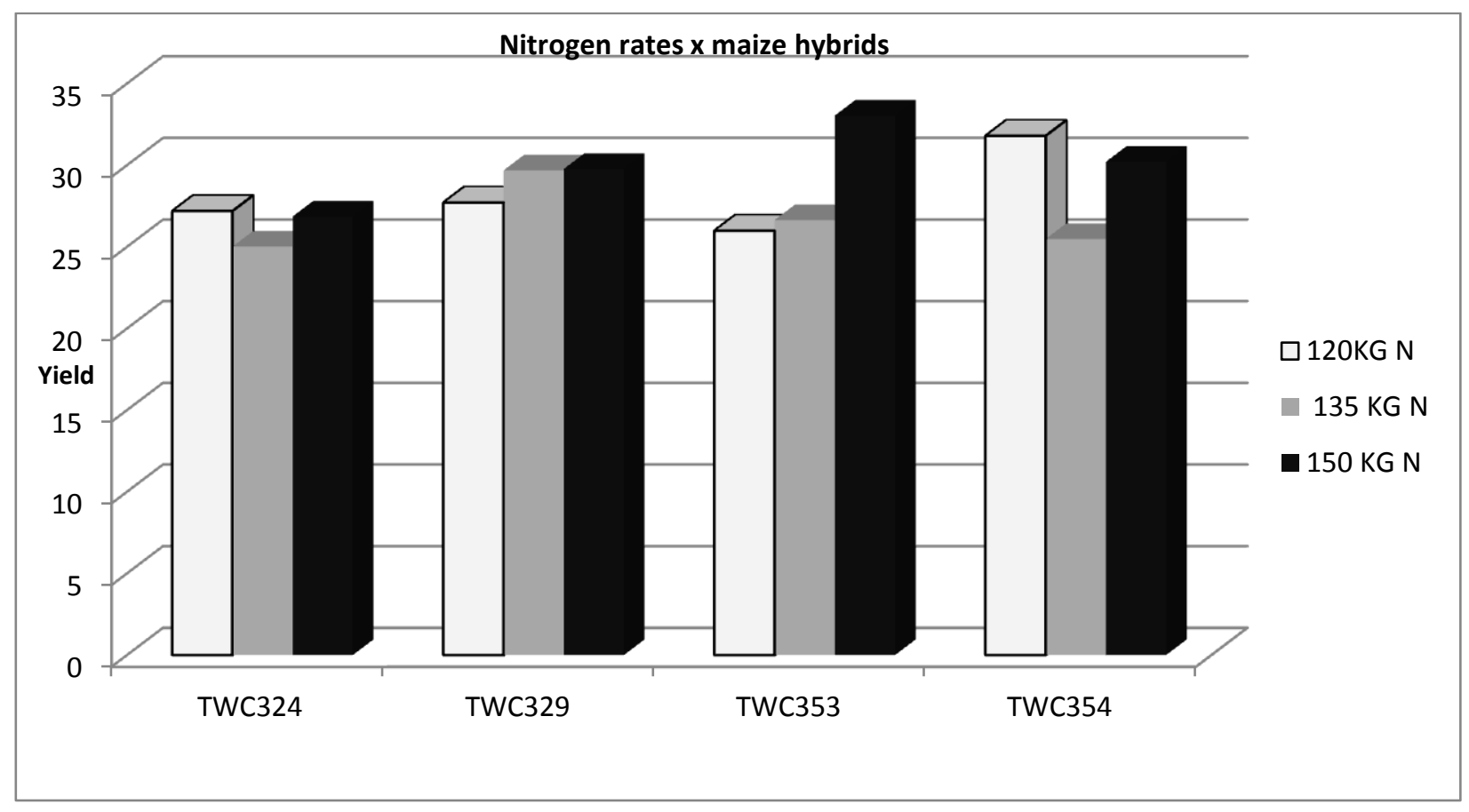

Fig. (2): Effect of interaction between nitrogen fertilization and maize hybrids on grain yield at Mallawy location over two seasons. 


\subsection{Simple correlation}

At Gemmeiza location the simple correlation Coefficients between each two traits were calculated in the combined analysis across years. The association between grain yield $\mathrm{fed}^{-1}$ and its related traits in maize plants gives very useful information for the plant breeder who wants to incorporate desirable traits. Table (6) shows highly significant and positive correlation values between grain yieldfed ${ }^{-1}$, plant height, ear height, ear length, and 100 kernel weight. Meanwhile highly significant and positive correlation values were detected between ear diameter and each of plant was a positive and highly significant association between ear length and each of plant height, ear height, and ear length. Also, there height and ear height. Kernels per row had highly significant and positive correlation with ear length. Moreover, highly significant and negative correlation values were detected between 100 kernel weight and each of ear diameter and number of rows per ear. These results are in agreement with those obtained by El-Badawy and Mehasen (2011) and Mehasen et al. (2015).

These findings indicate that indirect selection for each or both plant height, ear height, ear length, and 100 kernel weight are accompanied by high yielding and more effective for the improvement of grain yield in maize. These results are in agreement with those obtained by Eraky et al., (1983) and Mehasen et al. (2015). plant height, ear height, ear length and kernels /row. These results are in line with those reported by Khodarahmpour and Hamidi (2012) and Zamaninejad et al. (2013).

\subsection{Factor analysis}

At Gemmeiza location, the factor analysis technique divided the nine studied traits into three independent groups or factors which explained $69.262 \%$ of the total variability in the dependence structure. The factors were constructed by applying the principal component approach to establish the dependent relationship between yield attributes in maize. For interpretation only, factor loadings greater than 0.5 were considered important (Seiler and Stafford, 1985). Summary of the composition of variables of the three extracted factors with loadings are given in Table (8). Factor 1 included three variables which accounted for $27.262 \%$ of the total variability. The three variables were the plant height, ear height, and grain yield. The three variables had high communality with factor 1 .

Factor 2 was made up of the number of ears per plot, ear diameter, rows per ear and 100 kernel weight. The sign of the loading values indicates the direction of the relationship between the factor and its related traits. It accounted for $25.289 \%$ of the total variability in the dependence structure.

Factor 3 was responsible for $16.711 \%$ of the total variability in the dependence structure. It

Table (6): Correlation coefficients between yield and yield attributes over maize hybrids and over seasons at Gemmeiza location.

\begin{tabular}{|l|c|c|c|c|c|c|c|c|c|}
\hline Characters & X1 & X2 & X3 & X4 & X5 & X6 & X7 & X8 & Y \\
\hline Plant height X1 & 1 & & & & & & & & \\
\hline Ear height X2 & $0.89^{* *}$ & 1 & & & & & & & \\
\hline N. ears /plot X3 & $-0.21^{* *}$ & -0.17 & 1 & & & & & & \\
\hline Ear length X4 & $0.44^{* *}$ & $0.38^{* *}$ & -0.09 & 1 & & & & & \\
\hline Ear diameter X5 & $0.37^{* *}$ & $0.29^{* *}$ & $-0.42^{* *}$ & $0.23^{*}$ & 1 & & & & \\
\hline Row/ear X6 & $-0.25^{* *}$ & $-0.28^{* *}$ & -0.09 & $-0.20^{*}$ & $0.37^{* *}$ & 1 & & & \\
\hline Kernel/row X7 & 0.13 & 0.11 & 0.16 & $0.48^{* *}$ & -0.45 & $-0.29^{* *}$ & 1 & & \\
\hline Kernel weight X8 & -0.04 & -0.08 & $0.27^{* *}$ & -0.03 & $-0.49^{* *}$ & $-0.37^{* * *}$ & 0.15 & 1 & \\
\hline Yield Y & $0.32^{* * *}$ & $0.29^{* *}$ & 0.19 & $0.36^{* *}$ & -0.09 & $-0.49^{* *}$ & 0.12 & $0.29^{* * *}$ & 1 \\
\hline
\end{tabular}

At Mallawy, the estimates of simple correlation coefficients for all comparisons between the studied traits are presented in Table (7). Grain yield had a positive and significant correlation with the number of ears /plot, ear length, kernels/row and kernel weight. Also, there was a positive and highly significant association between kernel weight and each of included the two characters ear length and kernels per row. These results are in agreement with Mehasen and Al-Fageh (2004). Bharathiveeramani and Prakash (2012) found that using Factor Analysis by plant breeders has the potential of increasing the comprehension of the casual relationship of variables and can help to determine the nature and sequence of traits to 
Table (7): Correlation coefficients between yield and yield attributes over maize hybrids and over seasons at Mallawy location.

\begin{tabular}{|l|c|c|c|c|c|c|c|c|c|}
\hline \multicolumn{1}{|c|}{ Characters } & $\mathbf{X 1}$ & $\mathbf{X 2}$ & $\mathbf{X 3}$ & $\mathbf{X 4}$ & $\mathbf{X 5}$ & $\mathbf{X 6}$ & $\mathbf{X 7}$ & $\mathbf{X 8}$ & Y \\
\hline Plant height X1 & 1 & & & & & & & & \\
\hline Ear height X2 & 0.89 & 1 & & & & & & & \\
\hline N. ears /plot X3 & -0.17 & -0.03 & 1 & & & & & & \\
\hline Ear length X4 & $0.25^{* *}$ & $0.30^{* *}$ & 0.07 & 1 & & & & & \\
\hline Ear diameter X5 & -0.10 & -0.18 & $-0.23^{*}$ & $0.23^{*}$ & 1 & & & & \\
\hline Rows/ear X6 & -0.11 & -0.07 & -0.09 & 0.08 & $0.44^{* *}$ & 1 & & & \\
\hline Kernel/row X7 & $0.26^{* *}$ & $0.34^{* *}$ & 0.18 & $0.56^{* *}$ & -0.05 & 0.11 & 1 & & \\
\hline 100 Kernel weight X8 & $0.48^{* * 1}$ & $0.39^{* *}$ & 0.18 & $0.48^{* *}$ & 0.02 & -0.14 & $0.29 * *$ & 1 & \\
\hline Grain yield Y & 0.11 & 0.18 & $0.63^{* *}$ & $0.35^{* *}$ & -0.01 & -0.02 & $0.34^{* *}$ & $0.47 * *$ & 1 \\
\hline
\end{tabular}

Table (8): Summary of factor loadings for nine traitss of maize at Gemmeiza location over two seasons.

\begin{tabular}{|c|c|c|c|c|}
\hline Variable & Loading & $\%$ Communality & Eigen values & $\%$ of variance \\
\hline Factor 1 & & & \multirow{4}{*}{2.454} & \multirow{4}{*}{27.262} \\
\hline Plant height & 0.911 & 0.872 & & \\
\hline Ear height & 0.898 & 0.830 & & \\
\hline Grain yield & 0.539 & 0.570 & & \\
\hline \multicolumn{3}{|l|}{ Factor 2} & \multirow{5}{*}{2.276} & \multirow{5}{*}{25.289} \\
\hline Number of ears /plot & 0.564 & 0.415 & & \\
\hline Ear diameter & -0.797 & 0.730 & & \\
\hline Rows/ear & -0.647 & 0.644 & & \\
\hline Kernels weight & 0.763 & 0.583 & & \\
\hline \multicolumn{3}{|l|}{ Factor 3} & \multirow{3}{*}{1.504} & \multirow{3}{*}{16.711} \\
\hline Ear length & 0.758 & 0.752 & & \\
\hline Kernels/row & 0.900 & 0.837 & & \\
\hline Cumulative variance & & & & 69.262 \\
\hline
\end{tabular}

be selected in a breeding program.

At Mallawy location, the factor analysis technique divided the nine studied traits into three independent groups or factors which explained $73.516 \%$ of the total variability in the dependence structure. Summary of the composition of variables of the three extracted factors with loadings are given in Table (9). Factor 1 included three variables which accounted for $28.173 \%$ of the total variability. The three variables were plant height, ear height and 100 kernel weight. The three variables had high communality with factor 1 .

Factor 2 was made up of the number of ears/plot, ear length, kernels per row and grain yield. The sign of the loading values indicates the direction of the relationship between the factor and its related traits. It accounted for $24.759 \%$ of the total variability in the dependence structure.

Factor 3 was responsible for $20.584 \%$ of the total variability in the dependence structure. It included the two characters ear diameter and rows per ear. 
Table (9): Summary of factor loadings for nine traits of maize at Mallawy location over two seasons.

\begin{tabular}{|c|c|c|c|c|}
\hline Variable & Loading & $\%$ Communality & Eigen values & $\%$ of variance \\
\hline Factor 1 & & & \multirow{4}{*}{2.536} & \multirow{4}{*}{28.173} \\
\hline Plant height & 0.942 & 0.897 & & \\
\hline Ear height & 0.910 & 0.830 & & \\
\hline 100 Kernel weight & 0.633 & 0.637 & & \\
\hline Factor 2 & & & \multirow{5}{*}{2.228} & \multirow{5}{*}{24.759} \\
\hline Number of ears/plot & 0.817 & 0.771 & & \\
\hline Ear length & 0.488 & 0.660 & & \\
\hline Kernels/row & 0.542 & 0.662 & & \\
\hline Yield & 0.876 & 0.785 & & \\
\hline Factor 3 & & & \multirow{3}{*}{1.853} & \multirow{3}{*}{20.584} \\
\hline Ear diameter & 0.824 & 0.706 & & \\
\hline Rows/ear & 0.798 & 0.668 & & \\
\hline Cumulative variance & & & & 73.516 \\
\hline
\end{tabular}

\section{Conclusion}

It could be recommended that fertilizing TWC353 maize hybrid by $150 \mathrm{~kg} \mathrm{~N}^{-1} \mathrm{fed}^{-1}$ could achieve the highest grain yield at two locations. Highly significant and positive associations were obtained between grain yield ardabfed ${ }^{-1}$ and each of plant height, ear height, ear length, and 100 kernel weight at Gemmeiza location. While at Mallawy location, grain yield had a positive and significant correlation with the number of ears per plot, ear length, kernels/row and 100- kernel weight. Factor analysis classified the nine studied traits, as grain yield components, into three main factors explained 69.26 and $73.516 \%$ of the total variability in the dependent structure at Gemmeiza and Mallawy locations, respectively. using of factor analysis by plant breeders has the potential of increasing the comprehension of the casual relationship of variables and can help to determine the nature and sequence of traits to be selected in a breeding program.

\section{REFERENCES}

Akmal M., Rahman H.U., Asim F.M. and Akbar H. (2010). Response of maize varieties to nitrogen application for leaf area profile, crop growth, yield and yield components. Pak. J. Bot., 42(3): 1941-1947.

Atia A.A.M. and Mahmoud A. A. (2006). Economic study to evaluate the nitrogen response curve in maize. J. Agric. Sci. Mansoura Univ.,31 (4):1833:1842.

Bharathiveeramani B. and Prakash M. (2012).
Factor analysis for yield contributing traits in maize (Zea mays L.). Electronic J. Plant Breed., 3(4): 998-1001.

Bundy L.G., Andraski T.W., Ruark M.D., Peterson A.E. (2011). Long-term continuous maize and nitrogen fertilizer effects on productivity and soil properties. Agron. J., 103: 1346-1351. doi: 10. 2134/agronj2011.0094

Cattel R. B. (1965). Factor analysis: an introduction to essential. I- The purpose and underlying models. Biometrics, 21: 195-215.

Ding L., Wang K.J., Jiang G.M., Biswas1 D.K., Xu H., Li L.F. and Li Y.H. (2005). Effects of nitrogen deficiency on photosynthetic traits of maize hybrids released in different years. Ann. Bot., 96: 925-930.

El-Badawy M. E. M. and Mehasen S.A.S. (2011). Multivariate analysis for yield and its components in maize under zinc and nitrogen fertilization levels. Aust Basic. J.\& Appl. Sci., 5(12): 3008-3015.

ErakyA.G., Salem A.H., Kamel N.M. and Gouda A.Sh.A. (1982). Effect of some agronomic practices on certain growth criteria in maize. Fac. Agric. Zagazig Univ., Res. Bull.,732.

Eraky A.G., A. H. Salem and A. Sh. A. Gouda (1983). The interrelationships between growth and yield attributes in maize .proceeding of the first conference of agronomy. Volume 1-(A) cereal crops: 95-110. 
Gouda A. Sh. A., El-Galfy A. M. K.and Hassan M.M.M. (2009). Response of some white maize single crosses to nitrogen fertiliz. Egypt. J. Appl.Sci.,24(11):148-163.

Gouda A.Sh.A.(1997).Response of yellow maize hybrids to nitrogen fertilization under different environmental conditions. Egypt. J. Appl.Sci;12(12):492-504.

Gouda A.Sh.A., Balba M. M.A. and Faisl R.I.I. (1992). Response of some maize varieties to nitrogen fertilization . Ann. Agric. Sci., Moshtohor .Zagazig Univ., 30(4):16511663.

Gomez K. A. and Gomez A. A. (1984). Statistical Procedures For Agricultural Research. $2^{\text {nd }}$ Ed. John Wiley \& Sons, Inc. NewYork, USA, 680p.

Kaiser H. F. (1958). The Varimax critertion for analytic notation in factor analysis. Psychometrica, 23:187-200.

Kandil E.E.E. (2013). Response of some maize hybrids (Zea mays L.) to different levels of nitrogenous fertilization. Appl. Sci. Res., 9(3):1902-1908.

Khodarahmpour Z. and Hamidi J. (2012). Study of yield and yield components of corn (Zea mays L.) inbred lines to drought stress. Afr. J. Biotech., 11(13), pp. 30993105.

Levene H. (1960). Robust tests for equality of variances. In Ingram Olkin, Harold Hotelling, Etalia, Stanford University press. USA, Santa Clara country, Clifornia pp. 278-292.

Majid M. A., Saiful Islam M. El Sabagh A., Hasan M.K., Saddam M.O., Barutcular C., Ratnasekera D., Abdelaal Kh. A.A. and Islam M.S. (2017). Influence of varying nitrogen levels on growth, yield and nitrogen use efficiency of hybrid maize (Zea mays). J. Exper. Biol. and Agric. Sci.,- 5(2)134-142

Mehasen S.A.S. and Al-Fageh F. M. (2004). Evaluation of growth, yield and its component of six yellow maize hybrids at different planting densities. Arab Univ. J. Agric. Sci., Ain Shams Univ., 12 (2): 569583.
Mehasen S.A.S. and El-Gizawy N.Kh. (2010). Evaluation of some maize varieties to soil moisture stress. Proc. 12th conf. Agron., Suez canal Univ., EL-Aresh, Egypt, 26-38 ( Sept., 20-22).

Mehasen S. A. S., El-Sarag Eman and Abdullah S. Sh. (2015). Evaluation of some maize hybrids under nitrogen fertilization levels Int'1 J. Sci., 5:6-14.

Mitiku W. and Asnakech H. (2016) effect of nitrogen fertilizer on growth, yield and yield components of maize (Zea mays 1.) in decha district, southwestern Ethiopia. International Journal of research granhaalayah Vol.4 (Iss.2) 95-100

Nagabhushan N. M., Mallikarjuna C. H., Shashibhaskar M. S. and Prahalada G. D. (2011). Genetic variability and correlation studies for yield and related characters in single cross hybrids of maize (Zea mays L.). Curr. Biot., 5: 157-163.

Nzuve F., Githiri S., Mukunya D.M. and Gethi J. (2014). Genetic variability and correlation studies of grain yield and related agronomic traits in maize., J. Agric. Sci.,6(9):166-176.

Page A. L., Miller R. H. and Keeney D. R. (1982)."Methods of Soil Analysis", Part I: "Soil Physical Analysis", and Part II: "Chemical and Microbiological Properties". Soil Sci. Amer. Madison, Wisconsin, U.S.A.

Russell E.W. (1973). Soil Conditions and Plant Growth. 10th edition. London, UK.

Sieler G.I. and Stafford R. E. (1985). Factor analysis of components in guar. Crop Sci., 25: $905-908$.

Snedecor G.W. and Cochran G. W. (1989). Statistical Methods, $8^{\text {th }}$ Ed. Iowa State Univ. Press, Ames, Iowa, USA.

Zamaninejad M., Khorasani S. Kh., Moeini M. J. and Heidarian A. R. (2013). Effect of salicylic acid on morphological characteristics, yield and yield components of Corn (Zea mays L.) under drought condition. Europ. J. Experi. Biol., 3(2):153-161. 
إستخدام الارتباط وتقليل عدد العوامل لاراسة استجابة الهجن الثلاثية للأرة الشامية للمعدلات المتزايدة

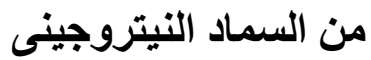

محمود بيومى عبد الجواد الكومى - مها جلال محمد بلبع - أشرف كمال مصطقى ـ هدى السيد العربى ابراهيم"

قسم بحوث الذرة الثامية ـ معهد بحوث المحاصيل الحقلية ـ * المعمل المركزي لبحوث التصميح و التحليل الاحصائى ـ

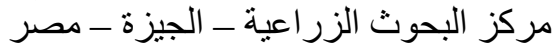

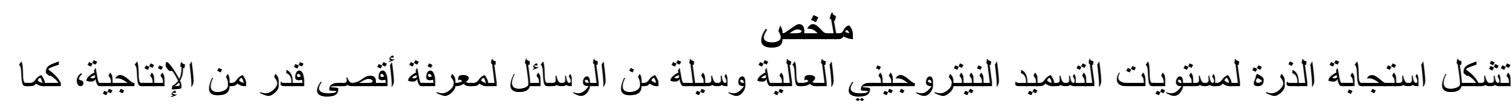

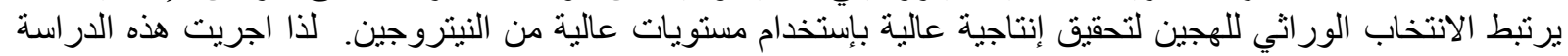

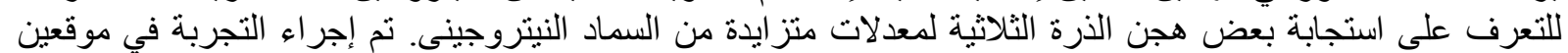

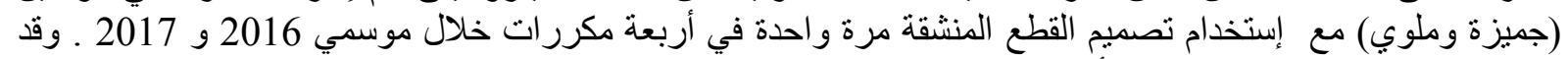

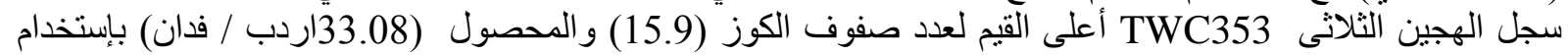

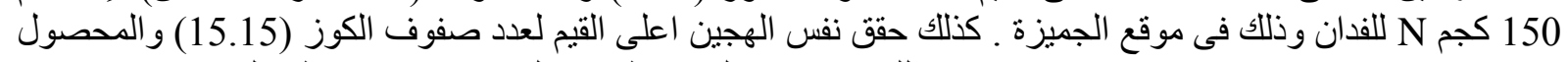

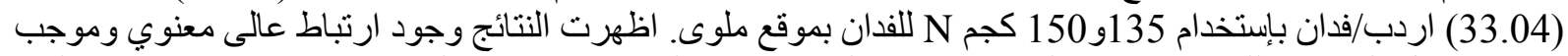

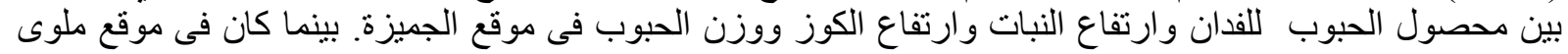

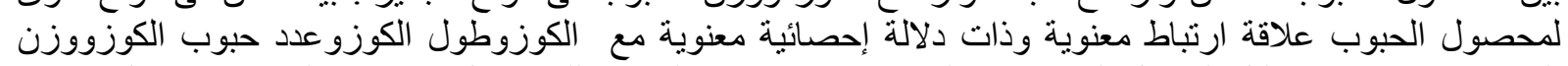

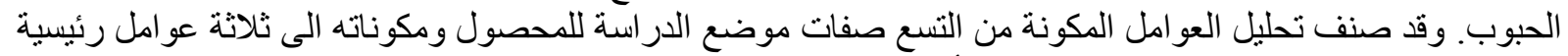

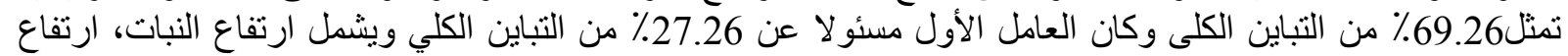

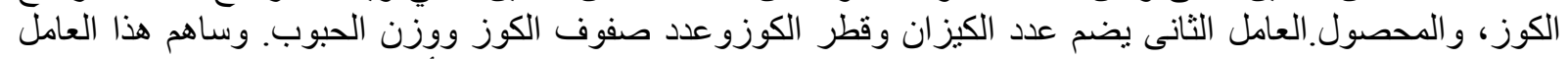

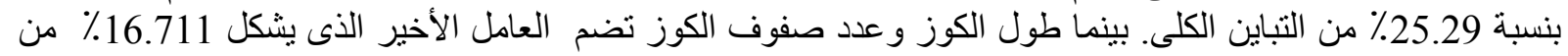

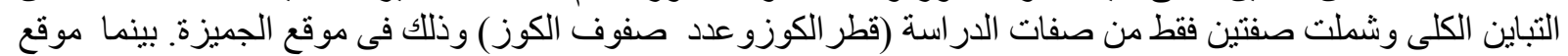

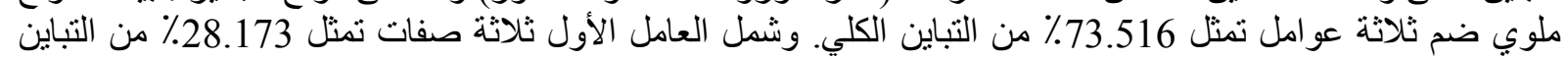

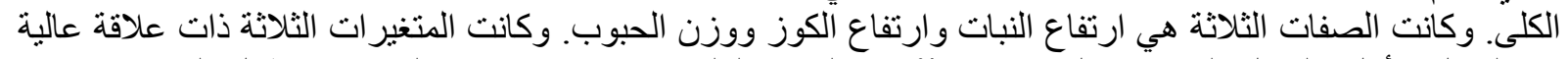

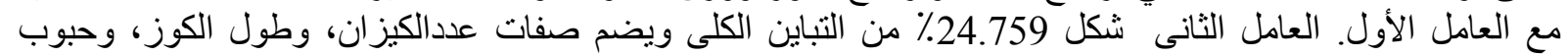

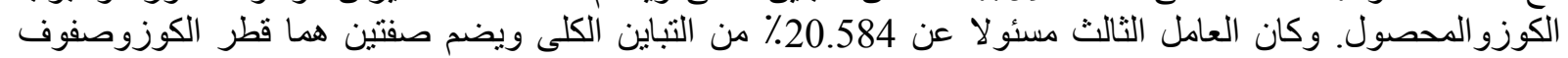
الكوز.

المجلة العلمية لكلية الزراعة - جامعة القاهرة - المجلد (69) العدد الاول (يناير 2018):73- 84. 